\title{
Atypical Complete Femoral Fractures Associated with Bisphosphonate Use or Not Associated with Bisphosphonate Use: Is There a Difference?
}

\author{
Sang-Min Kim, ${ }^{1}$ Youn-Soo Park, ${ }^{2}$ Young-Wan Moon, ${ }^{2}$ Seung-Hoon Kang, ${ }^{3}$ Ingwon Yeo, ${ }^{2}$ \\ Seung-Min Oh, ${ }^{1}$ and Seung-Jae Lim $^{2}$ \\ ${ }^{1}$ Department of Orthopaedic Surgery, Seoul Medical Center, Seoul, Republic of Korea \\ ${ }^{2}$ Department of Orthopaedic Surgery, Samsung Medical Center, Sungkyunkwan University School of Medicine, \\ Seoul, Republic of Korea \\ ${ }^{3}$ Department of Orthopaedic Surgery, Samsung Changwon Hospital, Sungkyunkwan University School of Medicine, \\ Changwon, Republic of Korea \\ Correspondence should be addressed to Seung-Jae Lim; limsj70@gmail.com
}

Received 25 August 2016; Accepted 23 October 2016

Academic Editor: Sheldon Lin

Copyright (c) 2016 Sang-Min Kim et al. This is an open access article distributed under the Creative Commons Attribution License, which permits unrestricted use, distribution, and reproduction in any medium, provided the original work is properly cited.

\begin{abstract}
The purpose of this study is to compare clinical characteristics and surgical outcome of atypical complete femoral fractures associated with bisphosphonates (BPs) use and those of fractures not associated with BPs use. Seventy-six consecutive patients (81 fractures) who had been operatively treated for a complete atypical femoral fracture were recruited. Of the 81 fractures, 73 occurred after BPs medication of at least 3 years (BP group) while 8 occurred without a history of BP medication (non-BP group). There were no differences in demographic data and fracture- and surgery-associated factors between the two groups. Of 76 patients (81 fractures), 54 (66.7\%) fractures showed bony union within 6 months after the index surgery and 23 (28.4\%) showed delayed union at a mean of 11.2 months (range, $8-18$ months). The remaining 4 fractures were not healed, even 18 months after the index surgery. There was no difference in healing rate between the BP group and the non-BP group. There were strong correlations between the fracture height and the degree of bowing regardless of BPs medication. All fractures except 1 occurred at the diaphyseal region of the femur when not associated with BP medication.
\end{abstract}

\section{Introduction}

Bisphosphonates (BPs) are known to inhibit osteoclastmediated bone resorption, which may cause accumulation of trabecular microdamage [1] and compromise the mechanical and regenerative properties of bone, resulting in fractures $[2,3]$. Long-term use of bisphosphonates (BPs) has been considered one of the causes of low-energy femoral fracture, and these have been termed atypical femoral fractures [3-9]. However, the association between long-term use of BPs and atypical femoral fractures is still unclear and lots of studies have showed variability of relative risk values [10-13].

In 2010, the American Society for Bone and Mineral Research (ASBMR) defined atypical femoral fractures with 5 major features including being located on subtrochanteric and diaphyseal region, medial spike or lateral cortex hypertrophy, transverse or short oblique configuration, and being not comminuted and after no or minimal trauma [14]. It was also noted that these fractures may occur even without use of BPs. ASBMR Task Force 2013 revised case definition of atypical femoral fractures, deleting the clause associated with the use of pharmaceutical agents such as BPs, glucocorticoids, and proton pump inhibitors [15]. Under this definition, stress fractures which have been well recognized to be caused by bowing deformity may also be classified as atypical femoral fractures [16].

Several recent studies have addressed the operative treatment, complications, and healing rates of atypical femoral fractures after BPs medication [7, 17-21]. Delayed or failed fracture healing is becoming a major concern after fracture 
stabilization in patients taking BPs [19]. However, there are limited data regarding atypical fractures occurring without use of BPs. Only a few cases have been reported to date. Therefore, this study was investigated to compare clinical characteristics and surgical outcome of atypical complete femoral fractures associated with BPs use and those of fractures not associated with BPs use.

\section{Materials and Methods}

From July 2008 to February 2014, a consecutive series of 86 patients (91 fractures) who had been operatively treated for a complete atypical femoral fracture were identified. Atypical femoral fractures were defined by characteristic radiographic findings according to the criteria of the 2013 ASMBR Task Force [15]. All fractures were located along the femur from just distal to the less trochanter to just proximal to the supracondylar flare and occurred with a history of minimal or no trauma. Minimal trauma was defined as a slip or fall from a standing height or less [22]. Of these 86 patients, 3 patients ( 3 fractures) who had a previous experience of fracture, infection, or surgery of the affected femur were excluded from this study, as were 2 patients ( 2 fractures) who had a history of corticosteroid use, hormonal treatment, rheumatoid arthritis, metabolic bone disease other than osteoporosis such as hyperparathyroidism, or paraneoplastic syndrome. No pathologic fractures-associated metastatic malignancies were found. Five patients ( 5 fractures) were lost to follow-up before bony union. The final cohort comprised 76 patients (81 fractures). Of the 81 fractures, 73 occurred after BPs medication of at least 3 years (BP group) while 8 occurred without a history of BP medication (non-BP group). Mean follow-up period was $32.4 \pm 9.4$ months (range, 12-62 months). This study was approved by our Institutional Review Board and informed consent was waived for this retrospective study. Prodromal symptoms developed in 21 (25.9\%) of the 81 fractures and the mean duration of symptoms was $5.3 \pm 2.3$ months (range, 1-8 months). All patients discontinued BP medications at the time of admission to our hospital after drug verification.

For each patient, we retrospectively searched detailed demographic data including age, affected side, sex, body mass index (BMI), and bone mineral density (BMD). Comorbidities and functional status [23] of the study population are also investigated. From our fracture database, characteristics of fracture and surgery that include injury mechanism, fracture location, fracture height [8], bilaterality, operation time, and the time interval from injury to operation were identified. Fracture location was categorized into two groups (subtrochanteric and diaphyseal). All of the fractures were fixed with an intramedullary nail.

Standing femoral AP radiographs with correct patellar forwarding with no rotation and lateral radiographs with the medial and lateral condyle of the femur overlapping were selected for data analyses. Preoperatively, femoral neck-shaft angle [24] and coronal and sagittal bowing of the femur were evaluated. Femoral bowing was measured using the curvature of the opposite femur as previously described [22]. In cases of bilateral fractures, bilateral plain radiographs before the second fracture were available. With respect to the reduction status of fractures, coronal alignment of the femur was defined as valgus, neutral, or varus and sagittal alignment was defined as flexion, neutral, or extension. Fracture healing was defined as bony bridging of 3 or 4 cortices on anteroposterior (AP) and lateral radiographs [25]. Fractures which showed bony union more than 6 months after the index surgery were considered to achieve delayed union. Nonunion is defined as persistence of the fracture 12 months after initial trauma without any tendency to progressive union in the previous 3 months [26].

2.1. Statistical Analyses. Basic descriptive statistical analyses were used to describe the study population. Values were subjected to averaging or percentages with use of Statistical Package for the Social Science software version 18.0 (SPSS, Chicago, IL, USA). The Mann-Whitney test was used for continuous variables, and Fisher exact test was used for categorical variables. Statistical significance was defined as $p \leq 0.05$. Spearman's correlation coefficient was used to examine the correlation between the fracture height and anterior or lateral femoral bowing.

\section{Results}

Demographic data of the BP group and the non-BP group are summarized in Table 1 . There were no significant differences between the two groups. Fracture- and surgery-associated factors of the BP and the non-BP groups are compared in Table 2. In the BP group, atypical fracture occurred at the subtrochanteric region in $27 / 73$ (36.9\%) while, in the non-BP group, only 1 of $8(12.5 \%)$ occurred at this region. All fractures except 1 occurred at diaphyseal region when not associated with BP medication. However, statistical significance was not seen between the two groups. Radiographic results before and after surgery are presented in Table 3. Preoperative femoral bowing and reduction configuration during surgery showed similar patterns between the two groups (Figure 1).

Of 76 patients (81 fractures) in the final cohort, $54(66.7 \%)$ fractures showed bony union within 6 months after the index surgery. Of the BP group (73 fractures) 49 achieved complete union within 6 months after surgery while of the non-BP group (8 fractures) 5 achieved complete union within 6 months after surgery $(p=1.000)$. Of 81 fractures, $23(28.4 \%)$ yielded delayed union at a mean of 11.2 months (range, 8-18 months), but there was no difference between the two groups (20/73 versus $3 / 8, p=0.682)$. The remaining 4 fractures were not healed, even 18 months after the index surgery. Of these 4 fractures, 2 received revision osteosynthesis (Figure 2) and 1 received total hip replacement; 1 patient refused reoperation up to the final follow-up. Among 8 of the non-BP group, 1 experienced refracture and underwent revision surgery. After she obtained complete union, implanted nail was removed. However, refracture occurred at the same region (Figure 3).

Correlation analysis results between the fracture height and the coronal/sagittal bowing are shown in Figure 4. There were positive correlations between the fracture height and the coronal/sagittal bowing in both the BP group $(r=0.728$ and 
TABLE 1: Baseline patient characteristics.

\begin{tabular}{|c|c|c|c|}
\hline & BP group $(n=73)$ & Non-BP group $(n=8)$ & $p$ \\
\hline Age (yr) & $72.6(58-85)$ & $73.4(61-84)$ & 0.698 \\
\hline Female sex & $73(100 \%)$ & $8(100 \%)$ & 1.000 \\
\hline Affected side (right: left) & $35: 38$ & $4: 4$ & 1.000 \\
\hline Body mass index $\left(\mathrm{kg} / \mathrm{m}^{2}\right)$ & $25.6(17.3-40.7)$ & $24.9(21.2-32.3)$ & 0.466 \\
\hline Bone mineral density ( $T$-score, femur) & $-2.2(-0.6--3.5)$ & $-2.3(-0.8--3.7)$ & 0.589 \\
\hline \multicolumn{4}{|l|}{ Comorbidities } \\
\hline Cardiovascular disease & $12(16.4 \%)$ & $2(25.0 \%)$ & 0.621 \\
\hline Cerebrovascular disease & $5(6.8 \%)$ & $1(12.5 \%)$ & 0.475 \\
\hline Chronic pulmonary disease & $8(11.0 \%)$ & $1(12.5 \%)$ & 1.000 \\
\hline Chronic renal disease & $1(1.4 \%)$ & $0(0.0 \%)$ & 1.000 \\
\hline Chronic liver disease & $5(6.8 \%)$ & $0(0.0 \%)$ & 1.000 \\
\hline Diabetes mellitus & $17(23.3 \%)$ & $2(25.0 \%)$ & 1.000 \\
\hline Smoking & $1(1.4 \%)$ & $0(0.0 \%)$ & 1.000 \\
\hline Parkinsonism & $1(1.4 \%)$ & $1(12.5 \%)$ & 0.189 \\
\hline Cognitive impairment & $3(4.1 \%)$ & $1(12.5 \%)$ & 0.346 \\
\hline ASA classification & & & 0.216 \\
\hline I or II & $66(90.4 \%)$ & $6(75.0 \%)$ & \\
\hline III or IV & $7(9.6 \%)$ & $2(25.0 \%)$ & \\
\hline Koval score & & & 1.000 \\
\hline 1 & $23(31.5 \%)$ & $3(37.5 \%)$ & \\
\hline 2 or 3 & $39(53.4 \%)$ & $4(50.0 \%)$ & \\
\hline$\geq 4$ & $11(15.1 \%)$ & $1(12.5 \%)$ & \\
\hline Presence of prodromal symptoms ${ }^{\dagger}$ & & & 0.673 \\
\hline Yes & $20(27.4 \%)$ & $1(12.5 \%)$ & \\
\hline No & $53(72.6 \%)$ & $7(87.5 \%)$ & \\
\hline
\end{tabular}

${ }^{\dagger}$ Prodromal symptoms before fracture, such as thigh pain.

TABLE 2: Characteristics of fracture and surgery.

\begin{tabular}{lcc}
\hline & BP group $(n=73)$ & Non-BP group $(n=8)$ \\
\hline Injury mechanism & & 0.722 \\
$\quad$ Fall-down & $33(45.2 \%)$ & $3(37.5 \%)$ \\
$\quad$ Slip-down & $35(47.9 \%)$ & $4(50.0 \%)$ \\
$\quad$ No trauma & $5(6.9 \%)$ & $1(12.5 \%)$ \\
Fracture location & $27(36.9 \%)$ & $1(12.5 \%)$ \\
$\quad$ Subtrochanteric & $46(63.1 \%)$ & $7(87.5 \%)$ \\
$\quad$ Diaphyseal & $41.0(17.2-66.3)$ & $46.5(20.1-54.2)$ \\
Fracture height (\%) & $18(24.7 \%)$ & $2(25.0 \%)$ \\
Fracture bilaterality & $55(75.3 \%)$ & 6.252 \\
$\quad$ Complete or incomplete fracture in the contralateral side & 1.580 \\
$\quad$ No fracture in the contralateral side & 4.000 \\
Operation time (min) & & $113.9 \pm 34.2(60-245)$ \\
The time interval from injury to operation (day) & & $4.3 \pm 1.6(1-10)$ \\
\hline
\end{tabular}




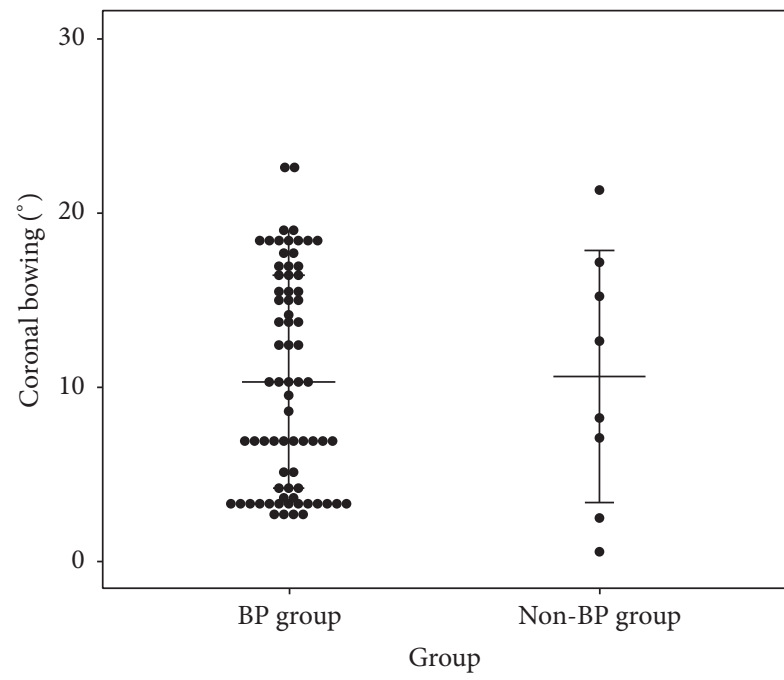

(a)

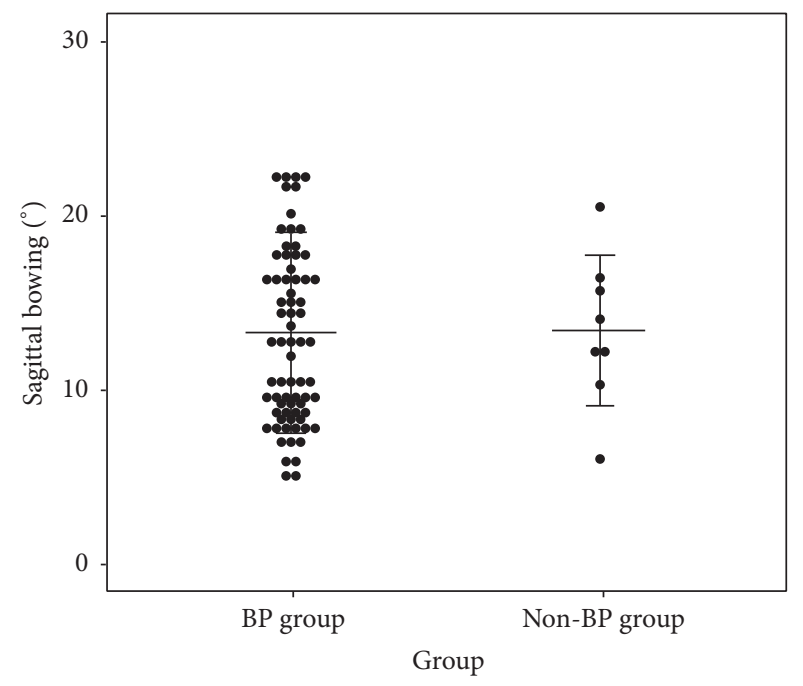

(b)

FIGURE 1: Comparison of (a) coronal and (b) sagittal bowing in patients of the BP group and the non-BP group. The mean, standard deviation, and data plots are shown.
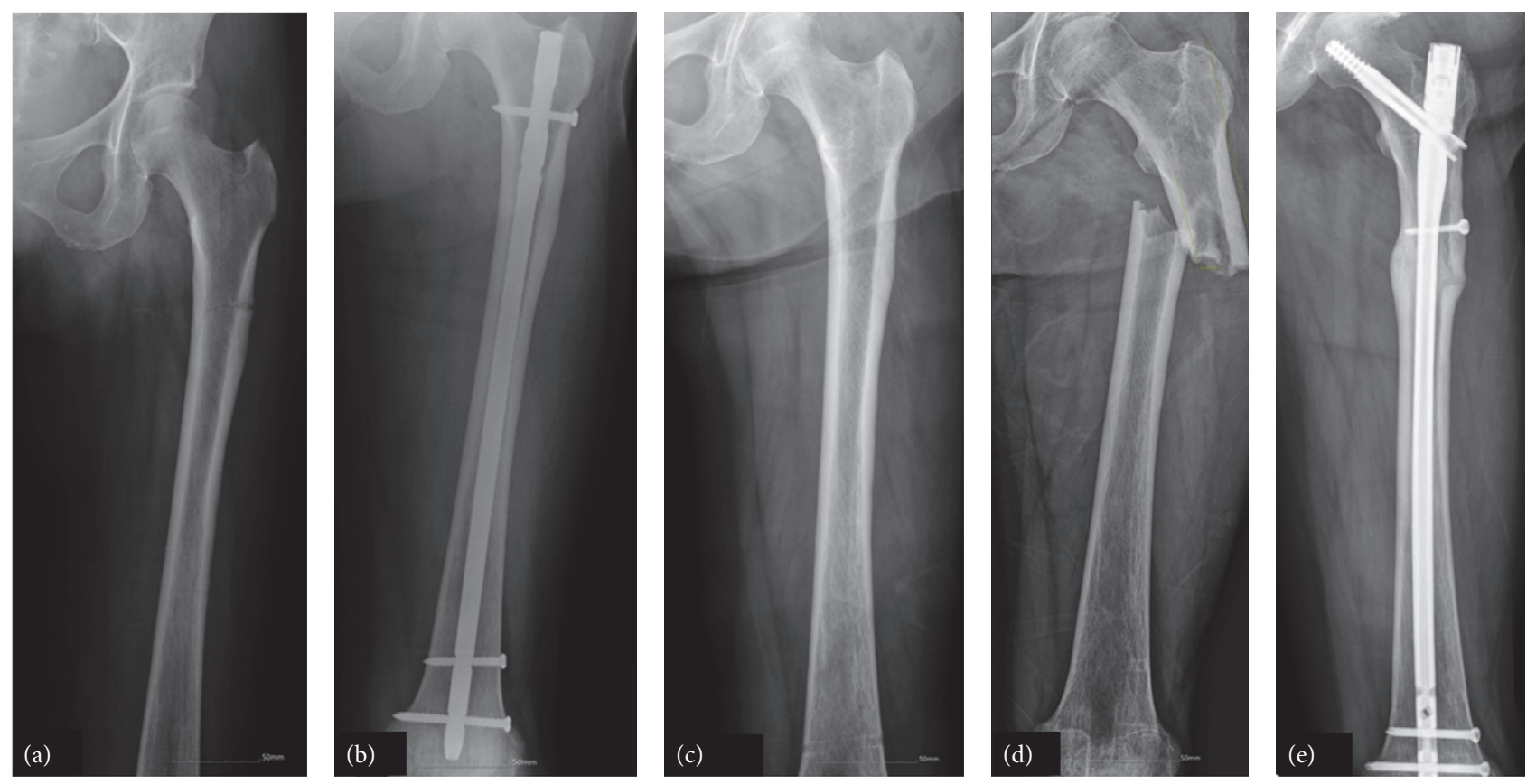

Figure 2: (a) Initial radiograph of a 64-year-old woman shows an atypical fracture of the subtrochanteric region of the femur. She had no history of antiosteoporotic medication. (b) Intramedullary nailing was performed. (c) After complete healing, implanted nail was removed 12 months after initial surgery. (d) Refracture occurred 33 months after nail removal. (e) A radiograph at 12 months after surgery shows complete healing.

$r=0.665$, resp.) and the non-BP group $(r=0.833$ and $r=$ 0.929 , resp.).

\section{Discussion}

After the concept of atypical femoral fractures became known widely, in Asian countries especially, stress fractures associated with bowing deformity have been confused with atypical fractures associated with long-term use of BPs [16]. According to ASMBR 2013, stress fractures, which occur without a history of BPs medication may also be classified as atypical femoral fractures. In our series, 68 patients (73 fractures) with a history of BPs medication of at least 3 years (BP group) and 8 patients (8 fractures) without a history of BP medication (non-BP group) are recruited. Tan et al. [27] reported the rate of atypical femoral fractures without BP use 

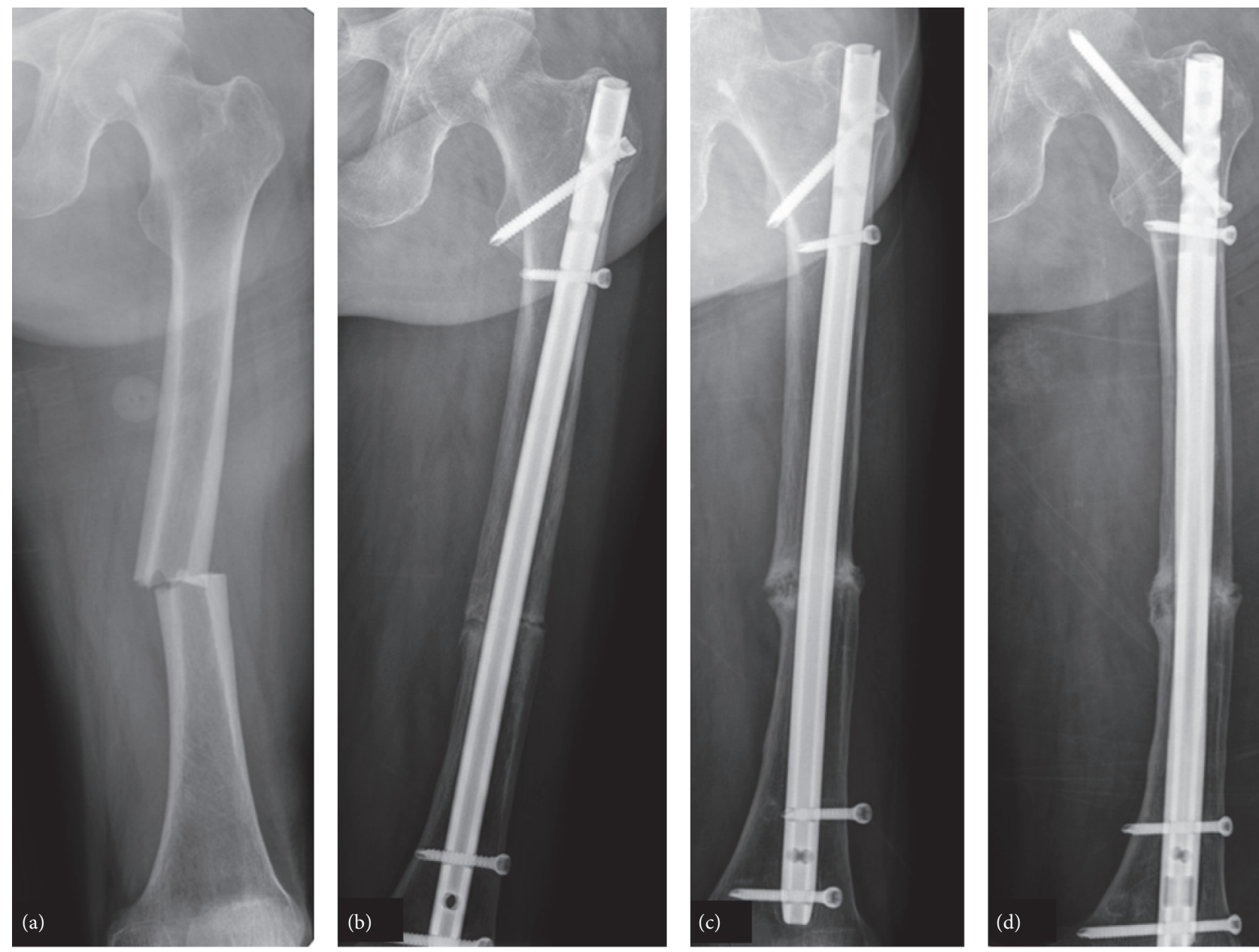

FIGURE 3: (a) A 77-year-old woman with a history of BP medication for 8 years experienced a complete femoral fracture after slip-down. (b) At 6 months after surgery, the fracture site was not healed. (c) She went on to develop nonunion with loosening of a distal interlocking screw even 3 years after surgery. (d) Revision surgery with a thicker and longer nail was performed.

was $8 \%$ in their series of 50 patients who fulfilled distinctive radiographic characteristics of the atypical fracture.

Recently, there is fast growth in knowledge regarding atypical fractures associated with long-term BP use and our improved understanding of them could be beneficial to prevent functional decline and deconditioning in the elderly. However, we have little information about atypical fractures occurring without a history of BPs medication. It is still unclear whether clinical characteristics and surgical outcome of atypical complete femoral fractures without a history of BP use are similar to those of fractures associated with long-term use of BPs. In the present study, there were no differences in demographic data and fracture- and surgery-associated factors between the two groups.

Of 76 patients ( 81 fractures) in the final cohort, 54 (66.7\%) fractures showed bony union within 6 months after the index surgery and $23(28.4 \%)$ showed delayed union at a mean of 11.2 months (range, 8-18 months). A previous study [18], which evaluated 41 atypical, low-energy femoral fractures associated with $\geq 5$ years of BP, reported that $98 \%$ (40/41) showed radiographic union at a mean of 8.3 months (range, 2-18 months). Healing times of almost 8 months for these fractures seemed to be longer than those for typical femoral fractures, which heal at an average of 3 to 6 months. Our findings also showed that there was no difference in healing time between the $\mathrm{BP}$ group and the non-BP group.

Asians have differences in femur geometry with higher rates of bowing, compared to western populations [5]. Some authors suggested that stress concentration on the diaphysis due to anteriorly and laterally bent shape of the femur may be an important causative factor of the atypical fracture $[8,16,22]$. Mechanical alterations due to the bowing of the femur increase the bending force on the anterior and lateral cortex. Moreover, the progression of this deformity shifts the area of maximal tensile stress to a more distal site of the diaphysis [8]. Our study demonstrated that there were strong correlations between the fracture height and the degree of bowing regardless of BPs medication. As the bowing deformity was larger, the site of the fracture in the femur was more distal. 


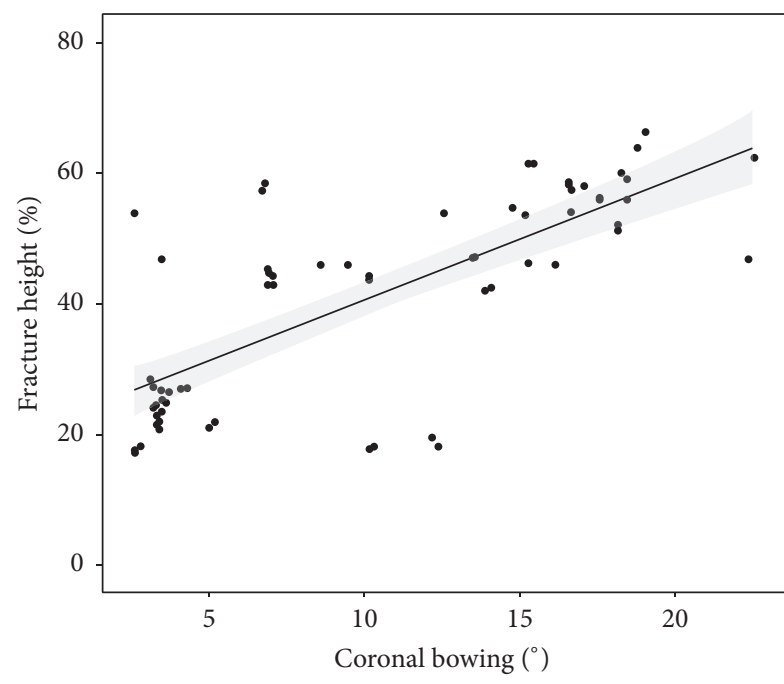

(a)

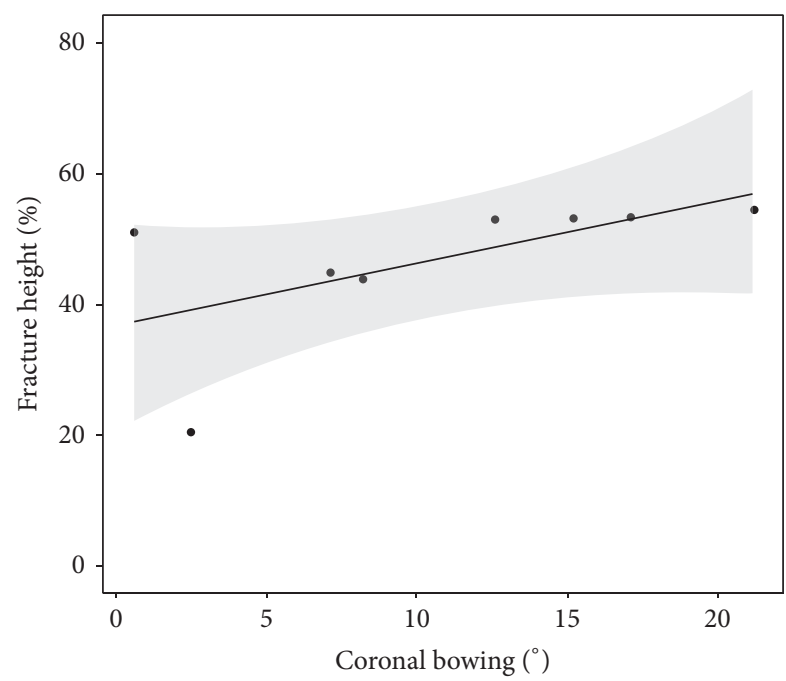

(c)

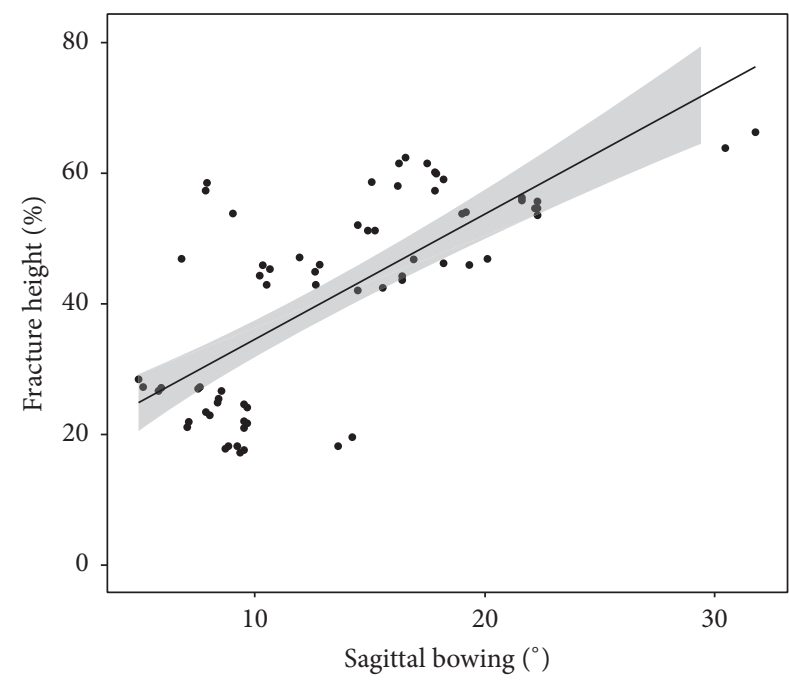

(b)

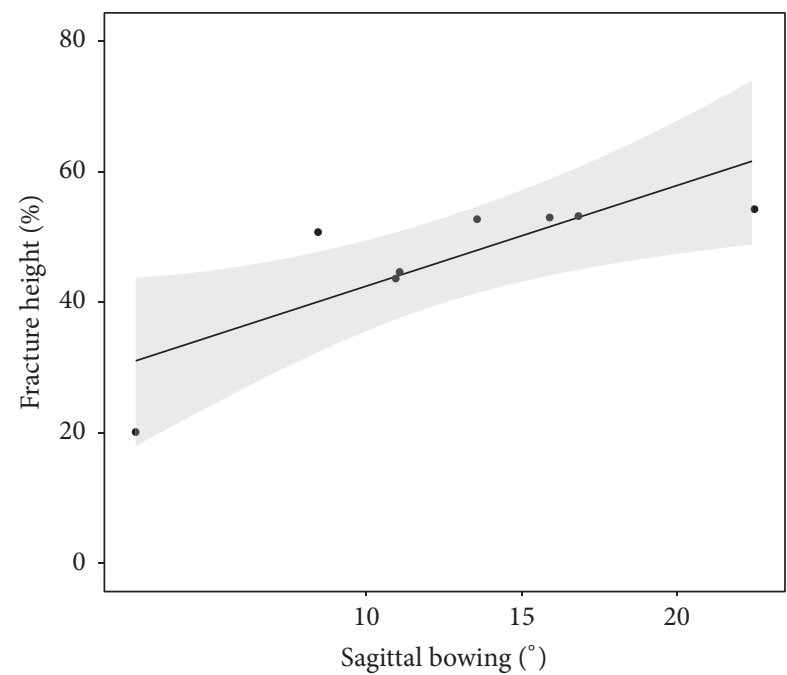

(d)

FiguRE 4: Scattered diagram showing the relation between the fracture height and the femoral bowing (a) in the coronal plane (Pearson's coefficient of 0.728 ) and (b) in the sagittal plane (0.665) of the BP group and (c) in the coronal plane (0.833) and (d) in the sagittal plane (0.929) of the non-BP group. Linear line; approximate line, gray zone; $95 \%$ prediction intervals.

The association between atypical femoral fractures and BPs therapy is controversial. BPs seem to increase risk of atypical fractures, with greater risk related to longer duration of management and declining risk after stopping BPs medication $[11,13]$. However, atypical fractures have also seen observed in patients who have never been exposed to BPs $[13,28]$ and our findings add the evidence to these findings. Some authors have demonstrated that bisphosphonates did not delay spine fusion as compared to controls $[29,30]$. Donnelly et al. [31] concluded that while BPs treatment may be an important risk factor for atypical fractures, it cannot be the sole risk factors. Thus, we believe that it is the nature of the fracture compared to the drug history that is more important.

This study has some limitations. First, this study was conducted with a retrospective nature, and therefore this design has inherent risk of observer bias, including the potential for missing data and inability to control confounding variables. Second, there were a limited number of patients. It is not easy to recruit a large sample size with a relatively rare injury. However, on investigation of the studies available regarding surgical outcome of such an injury, the current study is one of the largest ones. Third, we did not evaluate functional outcomes. Egol et al. [18] reported that $66 \%$ of patients with surgically treated complete atypical fractures became pain-free and pain combined with apprehension was a major cause of functional limitations. Lastly, the presence of osteomalacia may contribute to the findings of this study. More investigation on specific bone metabolism-related markers such as FGF23 level and associated genetic studies is not easily available under public health insurance system to which all of people should be affiliated. 
TABLE 3: Characteristics of radiographic results.

\begin{tabular}{|c|c|c|c|}
\hline & BP group $(n=73)$ & Non-BP group $(n=8)$ & $p$ \\
\hline \multicolumn{4}{|l|}{ Preoperative } \\
\hline Femoral neck-shaft angle $\left(^{\circ}\right)$ & $126.1(119-137.3)$ & $126(121.6-136)$ & 0.981 \\
\hline Coronal bowing of the femur $\left({ }^{\circ}\right)$ & $10.3(2.6-22.6)$ & $10.6(0.6-21.2)$ & 0.994 \\
\hline Sagittal bowing of the femur $\left(^{\circ}\right)$ & $13.3(4.9-31.8)$ & $13.4(6-20.5)$ & 0.758 \\
\hline \multicolumn{4}{|l|}{ Intraoperative } \\
\hline Reduction in the coronal plane & & & 0.828 \\
\hline Valgus & $9(12.3 \%)$ & $1(12.5 \%)$ & \\
\hline Neutral & $57(78.1 \%)$ & $6(75.0 \%)$ & \\
\hline Varus & $7(9.6 \%)$ & $1(12.5 \%)$ & \\
\hline Reduction in the sagittal plane & & & 0.628 \\
\hline Flexion & $2(2.8 \%)$ & $0(0.0 \%)$ & \\
\hline Neutral & $65(89.0 \%)$ & $7(87.5 \%)$ & \\
\hline Extension & $6(8.2 \%)$ & $1(12.5 \%)$ & \\
\hline \multicolumn{4}{|l|}{ Postoperative } \\
\hline Union within 6 months & $49(67.1 \%)$ & $5(63.5 \%)$ & 1.000 \\
\hline Delayed union & $20(27.4 \%)$ & $3(37.5 \%)$ & 0.682 \\
\hline Nonunion & $4(5.5 \%)$ & $0(0.0 \%)$ & 1.000 \\
\hline Reoperation & $3(4.1 \%)$ & $1(12.5 \%)$ & 0.346 \\
\hline Revision & $3(4.1 \%)$ & $1(12.5 \%)$ & 0.346 \\
\hline
\end{tabular}

In conclusion, of 76 patients (81 fractures), 54 (66.7\%) fractures showed bony union within 6 months after the index surgery and $23(28.4 \%)$ showed delayed union at mean of 11.2 months (range, $8-18$ months). There was no difference in healing rate between the BP group and the non-BP group. There were strong correlations between the fracture height and the degree of bowing both in the BP group and in the non-BP group. All fractures except 1 occurred at the diaphyseal region of the femur when not associated with BP medication.

\section{Competing Interests}

The authors declare that there is no conflict of interests regarding the publication of this paper.

\section{References}

[1] T. A. Einhorn, "The cell and molecular biology of fracture healing," Clinical Orthopaedics and Related Research, no. 355, supplement, pp. S7-S21, 1998.

[2] T. Mashiba, S. Mori, S. Komatsubara et al., "THe effects of suppressed bone remodeling by bisphosphonates on microdamage accumulation and degree of mineralization in the cortical bone of dog rib," Journal of Bone and Mineral Metabolism, vol. 23, no. 1, pp. 36-42, 2005.

[3] C. V. Odvina, J. E. Zerwekh, D. S. Rao, N. Maalouf, F. A. Gottschalk, and C. Y. C. Pak, "Severely suppressed bone turnover: a potential complication of alendronate therapy," Journal of Clinical Endocrinology and Metabolism, vol. 90, no. 3, pp. 1294-1301, 2005.

[4] B. Abrahamsen, "Bisphosphonate adverse effects, lessons from large databases," Current Opinion in Rheumatology, vol. 22, no. 4, pp. 404-409, 2010.
[5] J. S. Kang, Y. Y. Won, J. O. Kim et al., "Atypical femoral fractures after anti-osteoporotic medication: A Korean Multicenter Study," International Orthopaedics, vol. 38, no. 6, pp. 1247-1253, 2014.

[6] A. S. Neviaser, J. M. Lane, B. A. Lenart, F. Edobor-Osula, and D. G. Lorich, "Low-energy femoral shaft fractures associated with alendronate use," Journal of Orthopaedic Trauma, vol. 22, no. 5, pp. 346-350, 2008.

[7] J. W. Nieves and F. Cosman, "Atypical subtrochanteric and femoral shaft fractures and possible association with bisphosphonates," Current Osteoporosis Reports, vol. 8, no. 1, pp. 34-39, 2010.

[8] Y. Saita, M. Ishijima, A. Mogami et al., "The fracture sites of atypical femoral fractures are associated with the weight-bearing lower limb alignment," Bone, vol. 66, pp. 105-110, 2014.

[9] N. B. Watts and D. L. Diab, "Long-term use of bisphosphonates in osteoporosis," Journal of Clinical Endocrinology and Metabolism, vol. 95, no. 4, pp. 1555-1565, 2010.

[10] D. M. Black, M. P. Kelly, H. K. Genant et al., "Bisphosphonates and fractures of the subtrochanteric or diaphyseal femur," The New England Journal of Medicine, vol. 362, no. 19, pp. 1761-1771, 2010.

[11] R. M. Dell, A. L. Adams, D. F. Greene et al., "Incidence of atypical nontraumatic diaphyseal fractures of the femur," Journal of Bone and Mineral Research, vol. 27, no. 12, pp. 2544-2550, 2012.

[12] A. C. Feldstein, D. Black, N. Perrin et al., "Incidence and demography of femur fractures with and without atypical features," Journal of Bone and Mineral Research, vol. 27, no. 5, pp. 977-986, 2012.

[13] J. Schilcher, K. Michaëlsson, and P. Aspenberg, "Bisphosphonate use and atypical fractures of the femoral shaft," The New England Journal of Medicine, vol. 364, no. 18, pp. 1728-1737, 2011.

[14] E. Shane, D. Burr, P. R. Ebeling et al., "Atypical subtrochanteric and diaphyseal femoral fractures: report of a task force of the 
american society for bone and mineral Research," Journal of Bone and Mineral Research, vol. 25, no. 11, pp. 2267-2294, 2010.

[15] E. Shane, D. Burr, B. Abrahamsen et al., "Atypical subtrochanteric and diaphyseal femoral fractures: second report of a task force of the American Society for Bone and Mineral Research," Journal of Bone and Mineral Research, vol. 29, no. 1, pp. 1-23, 2014.

[16] Y. Oh, Y. Wakabayashi, Y. Kurosa, M. Ishizuki, and A. Okawa, "Stress fracture of the bowed femoral shaft is another cause of atypical femoral fracture in elderly Japanese: a case series," Journal of Orthopaedic Science, vol. 19, no. 4, pp. 579-586, 2014.

[17] S. S. Chan, Z. S. Rosenberg, K. Chan, and C. Capeci, "Subtrochanteric femoral fractures in patients receiving long-term alendronate therapy: imaging features," American Journal of Roentgenology, vol. 194, no. 6, pp. 1581-1586, 2010.

[18] K. A. Egol, J. H. Park, Z. S. Rosenberg, V. Peck, and N. C. Tejwani, "Healing delayed but generally reliable after bisphosphonate-associated complete femur fractures treated with IM nails," Clinical Orthopaedics and Related Research, vol. 472, no. 9, pp. 2728-2734, 2014.

[19] M. K. Grady, J. T. Watson, and L. K. Cannada, "Treatment of femoral fracture nonunion after long-term bisphosphonate use," Orthopedics, vol. 35, no. 6, pp. e991-e995, 2012.

[20] M. L. Prasarn, J. Ahn, D. L. Helfet, J. M. Lane, and D. G. Lorich, "Bisphosphonate-associated femur fractures have high complication rates with operative fixation," Clinical Orthopaedics and Related Research, vol. 470, no. 8, pp. 2295-2301, 2012.

[21] Y. A. Weil, G. Rivkin, O. Safran, M. Liebergall, and A. J. Foldes, "The outcome of surgically treated femur fractures associated with long-term bisphosphonate use," Journal of Trauma, vol. 71, no. 1, pp. 186-190, 2011.

[22] S. Sasaki, N. Miyakoshi, M. Hongo, Y. Kasukawa, and Y. Shimada, "Low-energy diaphyseal femoral fractures associated with bisphosphonate use and severe curved femur: a case series," Journal of Bone and Mineral Metabolism, vol. 30, no. 5, pp. 561567, 2012.

[23] K. J. Koval, G. B. Aharonoff, A. D. Rosenberg, R. L. Bernstein, and J. D. Zuckerman, "Functional outcome after hip fracture: effect of general versus regional anesthesia," Clinical Orthopaedics and Related Research, no. 348, pp. 37-41, 1998.

[24] R. M. Kay, K. A. Jaki, and D. L. Skaggs, "The effect of femoral rotation on the projected femoral neck-shaft angle," Journal of Pediatric Orthopaedics, vol. 20, no. 6, pp. 736-739, 2000.

[25] D. B. Whelan, M. Bhandari, M. D. McKee et al., "Interobserver and intraobserver variation in the assessment of the healing of tibial fractures after intramedullary fixation," The Journal of Bone \& Joint Surgery - British Volume, vol. 84, no. 1, pp. 15-18, 2002.

[26] D. Marsh, "Concepts of fracture union, delayed union, and nonunion," Clinical Orthopaedics and Related Research, no. 355, supplement, pp. S22-S30, 1998.

[27] S. C. Tan, S. B. J. Koh, S. K. Goh, and T. S. Howe, "Atypical femoral stress fractures in bisphosphonate-free patients," Osteoporosis International, vol. 22, no. 7, pp. 2211-2212, 2011.

[28] A. Giusti, N. A. T. Hamdy, O. M. Dekkers, S. R. Ramautar, S. Dijkstra, and S. E. Papapoulos, "Atypical fractures and bisphosphonate therapy: a cohort study of patients with femoral fracture with radiographic adjudication of fracture site and features," Bone, vol. 48, no. 5, pp. 966-971, 2011.

[29] S. M. Kim, W. Rhee, S. Ha, J. H. Lim, and I. T. Jang, "Influence of alendronate and endplate degeneration to single level posterior lumbar spinal interbody fusion," Korean Journal of Spine, vol. 11, no. 4, pp. 221-226, 2014.

[30] S. Ohtori, G. Inoue, S. Orita et al., "Comparison of teriparatide and bisphosphonate treatment to reduce pedicle screw loosening after lumbar spinal fusion surgery in postmenopausal women with osteoporosis from a bone quality perspective," Spine, vol. 38, no. 8, pp. E487-E492, 2013.

[31] E. Donnelly, A. Saleh, A. Unnanuntana, and J. M. Lane, "Atypical femoral fractures: epidemiology, etiology, and patient management," Current Opinion in Supportive and Palliative Care, vol. 6, no. 3, pp. 348-354, 2012. 


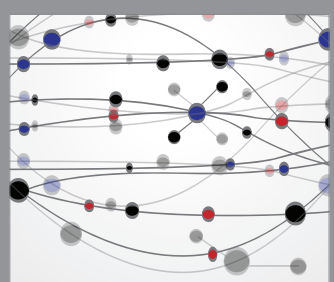

The Scientific World Journal
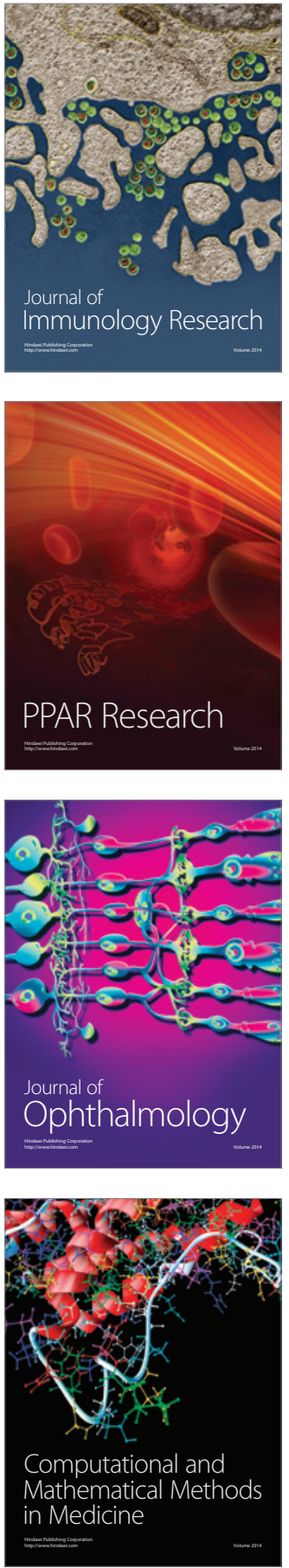

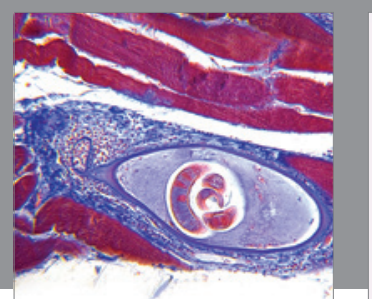

Gastroenterology Research and Practice

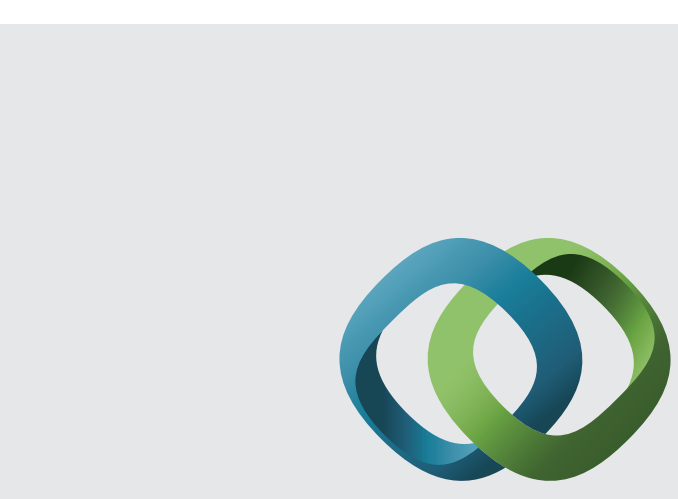

\section{Hindawi}

Submit your manuscripts at

http://www.hindawi.com
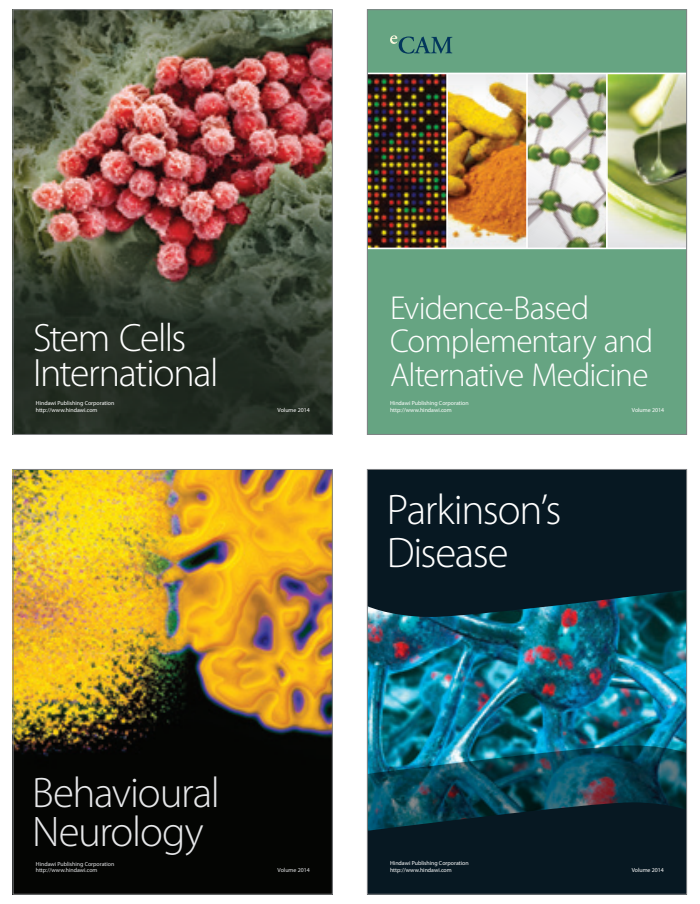
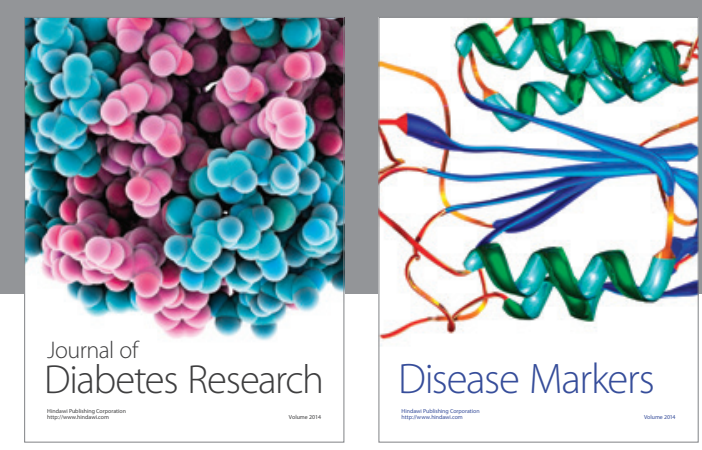

Disease Markers
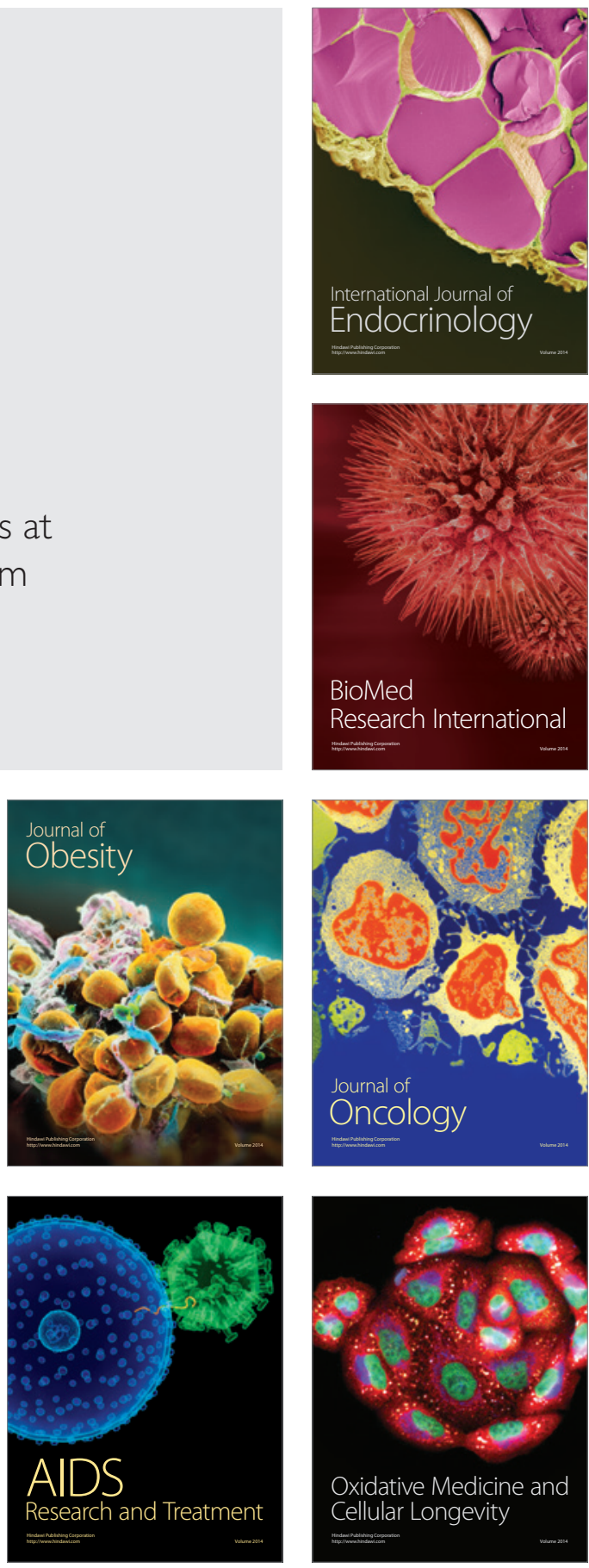